Int. J. Electrochem. Sci., 15 (2020) 10093 - 10103

\title{
An Electrochemical Fingerprint Approach for Direct Soy Sauce Authentic Identification Using a Glassy Carbon Electrode
}

\author{
Weihong $\mathrm{Wu}^{1, *}$, Qinwei Zhou ${ }^{1}$, Yuhong Zheng ${ }^{2}, \mathrm{Li} \mathrm{Fu}^{1, *}$, Jiangwei Zhu ${ }^{3}$ and Hassan Karimi-Maleh ${ }^{4}$ \\ ${ }^{1}$ College of Materials and Environmental Engineering, Hangzhou Dianzi University, Hangzhou \\ 310018, PR China \\ 2 Jiangsu Key Laboratory for the Research and Utilization of Plant Resources, Institute of Botany, \\ Jiangsu Province and Chinese Academy of Sciences, Nanjing 210014, P.R. China \\ ${ }^{3}$ Co-Innovation Center for Sustainable Forestry in Southern China, Nanjing Forestry University, \\ Nanjing, 210037, PR China \\ ${ }^{4}$ Department of Chemical Engineering, Laboratory of Nanotechnology, Quchan University of \\ Technology, Quchan, Islamic Republic of Iran \\ *E-mail: whwu@hdu.edu.cn; fuli@hdu.edu.cn
}

doi: $10.20964 / 2020.10 .69$

Received: 5 July 2020 / Accepted: 18 August 2020 / Published: 31 August 2020

An electrochemical technique for identifying soy sauce authenticity using a glassy carbon electrode is described. Due to the presence of salt in soy sauce, a three-electrode system can be directly inserted into soy sauce without the addition of electrolyte. The free amino acids and ATP-related compounds in soy sauce can be oxidized during differential pulse voltammetry (DPV) to form a fingerprint pattern. By using the presence/absence of peaks as criteria to calculate ratios, the DPV profiles can be used for identifying sixteen different soy sauces in this work. We believe this proposed methodology can be further extended to other food quality screening applications.

Keywords: Electrochemistry; Soy sauce; Glassy carbon electrode; Cluster analysis; Food safety ; Fingerprint

\section{FULL TEXT}

(C) 2020 The Authors. Published by ESG (www.electrochemsci.org). This article is an open access article distributed under the terms and conditions of the Creative Commons Attribution license (http://creativecommons.org/licenses/by/4.0/). 